observations on light microscopy. More interestingly, smaller crystals were seen pericellularly and in the intercellular matrix. Some crystals were seen adjacent to the chondrocytes and located in the lacuna (Fig. 1). Electron probe analysis of the crystals in various locations, and differing in size, gave a consistent calcium to phosphorus ratio of approximately $1 \cdot 15: 1$ similar to that of the calcium pyrophosphate standard. This ratio is quite different and much lower than the ratio of calcium and phosphorus in the three different types of apatite crystals found in human osteoarthritic articular cartilage. ${ }^{45}$

There were various non-specific changes in the cells and in the matrix components that were indicative of a rapidly degenerating cartilaginous tissue. Thus there were focal changes in the staining of the collagen and proteoglycan particles which gave the matrix, in some areas, a more granular appearance unlike the ordered, fibrous nature of normal cartilage matrix. In other areas there was a band-like, collagenous encapsulation of the lacuna area, which contained several cells in a cluster. Extracellular matrix vesicles were absent in many areas but weré sometimes seen pericellularly in focal areas where chondrocyte degeneration and necrosis was evident.

In terms of chondrocyte morphology two changes were particularly noticeable. Firstly, some cells showed abnormally large islands of glycogen in the cytoplasm (Fig. 1). Secondly, other chondrocytes showed abnormal increase in the amount of rough endoplasmic reticulum. If these observations are true it may imply increased synthesis of glycogen and other cartilage constituents. Inorganic pyrophosphate is a byproduct of many metabolic activities, and biosynthetic pathways, and this has been implicated by others as a mechanism for the formation of excess pyrophosphate in some tissues and fluids. From our observations we can only suggest that the calcium pyrophosphate crystals seen in the vicinity of chondrocytes may be formed by the interaction of a cellular product and some matrix ion or component. Further studies on the quantitative analysis of the level of pyrophosphatase-type enzymes in normal and degenerative cartilage are under way to see if the accumulation of pyrophosphate in the tissue is due to the lack of a specific degradative enzyme.

\section{References}

1 McCarty D J. Pseudogout. In: Hollander J L, McCarty D J, eds. Arthritis and allied conditions. Philadelphia: Lea and Febiger, 1972: 1140-60.

2 Bjelle A O, Sundstrom B K G. An ultrastructural study of the articular cartilage in calcium pyrophosphate dihydrate (CPPD) crystal deposition disease (chondrocalcinosis articularis). Calif Tiss Res 1975; 19: 63-71.

3 Schumacher H R. Ultrastructural findings in chondrocalcinosis and pseudogout. Arthritis Rheum 1976; 19: 413-25.

4 Ali S Y, Griffiths S. New types of calcium phosphate crystals in arthritic cartilage. Semin Arthritis Rheum 1981; 11, suppl 1: 124-6.

5 Ali S Y. New knowledge of osteoarthritis. $J$ Clin Pathol 1978; 31, suppl 12: 191-9.

\title{
Studies of pyrophosphate metabolism in relation to chondrocalcinosis
}

\author{
A. M. CASWELL, M. K. B. McGUIRE, AND R. G. G. RUSSELL \\ From the Department of Human Metabolism and Clinical Biochemistry, University of Sheffield Medical School, Sheffield \\ $S 102 R X$
}

In an attempt to understand the pathogenesis of chondrocalcinosis we have examined various aspects of the metabolism of inorganic pyrophosphate $\left(\mathrm{PP}_{\mathrm{i}}\right)$. Using a highly sensitive radiometric assay, ${ }^{1}$ we determined the concentration of $\mathrm{PP}_{\mathrm{i}}$ in serum and plasma and in cultured cells derived from normal individuals and from patients with chondrocalcinosis to identify any abnormality in $\mathrm{PP}_{\mathrm{i}}$ metabolism. We also examined the intracellular metabolism of $\mathrm{PP}_{\mathrm{i}}$ in cultured articular chondrocytes and meniscus cells derived from normal individuals to define the factors that influence the intracellular production and breakdown of $\mathrm{PP}_{\mathrm{i}}$. In addition, we studied the extracellular metabolism of $\mathrm{PP}_{\mathrm{i}}$ by these cells to define whether $\mathbf{P P}_{\mathrm{i}}$ can be produced outside cells and/or can cross the cell plasma membranes.

In agreement with the findings of earlier studies, ${ }^{23}$ we observed no differences in the serum and plasma concentrations of $\mathrm{PP}_{1}$ between normal individuals and patients with chondrocalcinosis. Similarly, in cultured skin fibroblasts, we failed to observe any difference in the content of $\mathrm{PP}_{\mathrm{i}}$ between cells derived from normal individuals and those derived from patients with chondrocalcinosis.
In contrast, the amounts of $\mathrm{PP}_{\mathrm{i}}$ in cultured articular chondrocytes and meniscus cells derived from patients with chondrocalcinosis were substantially higher than the $P P_{i}$ contents of these cell types derived from normal individuals, especially during primary culture. However, this may not necessarily reflect a change in intracellular $\mathrm{PP}_{\mathrm{i}}$ metabolism in these cell types in this condition, as it may be due to the continued presence of calcium pyrophosphate crystals that were present in vivo. For example, in one such culture derived from a patient with chondrocalcinosis crystals resembling triclinic calcium 
pyrophosphate dihydrate (CPPD) were observed in the cell layers and these slowly disappeared with time in culture. Moreover, medium samples obtained from cultures of articular chondrocytes and meniscus cells derived from two patients with chondrocalcinosis contained very high concentrations of $\mathrm{PP}_{\mathrm{i}}$ initially but these declined rapidly with time. Careful interpretation is therefore needed when apparently high concentrations of $\mathrm{PP}_{\mathrm{i}}$ are found in cultured articular chondrocytes and meniscus cells derived from patients with chondrocalcinosis, as these may simply reflect the continued presence of CPPD crystals that are trapped in the monolayer and are gradually washed away with each change of medium. These findings give little direct evidence for a specific increase in the $\mathrm{PP}_{\mathbf{i}}$ content of cells derived from patients with chondrocalcinosis, though it should be pointed out that none of our patients had recognisable hereditary forms of the disease.

In studies of the intracellular metabolism of $P P_{i}$, we have examined the influence of a variety of agents on cultured normal human articular chondrocytes and meniscus cells. We observed that increasing the medium phosphate concentration from 1 mmol to $2 \mathrm{mmol}$ or $10 \mathrm{mmol}$ in the presence of $1.3 \mathrm{mmol}$ calcium resulted in an increase in the intracellular $P_{i}$ content of chondrocytes.

In the presence of $10 \mathrm{mmol}$ phosphate, the intracellular $\mathrm{PP}_{\mathrm{i}}$ values were similar in the presence of 0.4 mmol, $1.3 \mathrm{mmol}$, or $1.8 \mathrm{mmol}$ calcium in the medium. When the calcium concentration was reduced to a low level, the intracellular $P_{P_{i}}$ concentration also fell. Treatment of chondrocytes or meniscus cells with oligomycin, an inhibitor of oxidative phosphorylation which promotes calcium influx into cells, increased their intracellular $P_{\mathrm{i}}$.

One potential intracellular source of $P_{P}$ is as a byproduct of the biosynthesis of glycosaminoglycans. We observed an increase in intracellular $\mathbf{P P}_{\mathbf{i}}$ in chondrocytes following treatment of the cells with the xyloside, 4-methylumbelliferyl$\beta$-D-xyloside, which stimulates glycosaminoglycan biosynthesis. Interestingly, the intracellular $P_{P}$ concentration of chondrocytes and meniscus cells also increased in the presence of tunicamycin, an inhibitor of glycosylation. This suggests that blocking of glycosylation and release of macromolecules from the cells might also impair the release of $\mathrm{PP}_{\mathrm{i}}$ from cells.

Taken together, these observations demonstrate that $\mathbf{P P}_{\mathbf{i}}$ concentrations in chondrocytes and meniscus cells can be altered by a variety of manipulations, which implies that the $\mathrm{PP}_{\mathrm{i}}$ concentrations of these cell types are under metabolic control and are influenced by the extracellular environment. The influence of the extracellular calcium concentration may be particularly important in relation to the pathogenesis of chondrocalcinosis and is of interest in relation to the known association between chondrocalcinosis and hyperparathyroidism. ${ }^{4}$

In studies of the extracellular metabolism of $\mathrm{PP}_{\mathrm{i}}$, we observed that added ${ }^{32} \mathrm{P}$-labelled $\mathrm{PP}_{\mathrm{i}}$ was only slowly removed from the medium on cultures of normal human articular chondrocytes such that about $20 \%$ of the added tracer was still present in the medium even after 48 hours Moreover, ${ }^{32} \mathrm{P}$-labelled orthophosphate $\left(P_{i}\right)$ in the medium appeared to account for a substantial fraction of the loss of label from $\mathbf{P P}_{\mathbf{i}}$. Hence it would appear that hydrolysis of $P P_{i}$ to $P_{i}$ is the major mechanism for the removal of $\mathrm{PP}_{\mathrm{i}}$ from the medium but that the ability of these cells to hydrolyse extracellular $\mathbf{P P}_{\mathrm{i}}$ is limited.

During short term incubation ( $1 \frac{1}{2}$ hours) of washed monolayers of normal human articular chondrocytes in medium without serum, we observed the release of small amounts of $\mathrm{PP}_{\mathrm{i}}$ into the medium, but the exact source of this $\mathrm{PP}_{\mathrm{i}}$ is not known. Much larger amounts of $\mathrm{PP}_{\mathrm{i}}$ were generated extracellularly following the addition of adenosine triphosphate (6.25-400 $\mu \mathrm{mol})$ to the medium without serum for $1 \frac{1}{2}$ hours. This effect is probably attributable to the action of a nucleoside triphosphate pyrophosphohydrolase present on the outside of the cells. This observation suggests that human articular chondrocytes possess a substantial capacity for the extracellular generation of $P P_{i}$. This mechanism might be important in the pathogenesis of chondrocalcinosis as it would provide a means of producing $\mathrm{PP}_{\mathrm{i}}$ outside cells in the presence of nucleoside triphosphates, which in turn might be derived by leakage from damaged cells in the neighbourhood.

\section{References}

1 Cheung C P, Suhadolnik R J. Analysis of inorganic pyrophosphate at the picamole level. Anal Biochem 1977; 83: 61.

2 Russell R G G, Bisaz S, Fleisch H, et al. Inorganic pyrophosphate in plasma, urine and synovial fluid of patients with pyrophosphate arthropathy (chondrocalcinosis or pseudogout). Lancet 1970; ii: 899-902.

3 Altman R D, Muniz O E, Pita J O, Howell D S. Articular chondrocalcinosis: microanalysis of pyrophosphate (PPi) in synovial fluid and plasma. Arthritis Rheum 1973; 16: 171-8.

4 Hamilton E B D. Diseases associated with calcium pyrophosphate deposition disease. Arthritis Rheum 1976; 19: 353-7. 